\title{
Ambulatory Status Over Time after Revascularization in Patients with Chronic Limb-Threatening Ischemia
}

\author{
Akio Kodama ${ }^{1}$, Mitsuyoshi Takahara ${ }^{2}$, Osamu lida ${ }^{3}$, Yoshimitsu Soga ${ }^{4}$, Hiroto Terashi ${ }^{5}$, Daizo Kawasaki ${ }^{6}$, \\ Yuichi Izumi ${ }^{7}$, Shinsuke Mii ${ }^{8}$, Kimihiro Komori ${ }^{1}$ and Nobuyoshi Azuma ${ }^{9}$ on behalf of the SPINACH investigators.
}

\author{
${ }^{1}$ Division of Vascular Surgery, Department of Surgery, Nagoya University School of Medicine, Nagoya, Japan \\ ${ }^{2}$ Department of Diabetes Care Medicine, and Department of Metabolic Medicine, Osaka University Graduate School of Medicine, \\ Osaka, Japan \\ ${ }^{3}$ Cardiovascular Center, Kansai Rosai Hospital, Hyogo, Japan \\ ${ }^{4}$ Department of Cardiology, Kokura Memorial Hospital, Fukuoka, Japan \\ ${ }^{5}$ Department of Plastic Surgery, Kobe University Graduate School of Medicine, Kobe, Japan \\ ${ }^{6}$ Cardiovascular Center, Morinomiya Hospital, Osaka, Japan \\ ${ }^{7}$ Nayoro City General Hospital, Hokkaido, Japan \\ ${ }^{8}$ Department of Vascular Surgery, Saiseikai Yahata General Hospital, Fukuoka, Japan \\ ${ }^{9}$ Department of Vascular Surgery, Asahikawa Medical University, Hokkaido, Japan
}

Aim: Maintaining functional status through revascularization is a major goal in patients with chronic limbthreatening ischemia (CLTI). Nevertheless, there is a lack of clarity on the impact of revascularization on mobility over time. This study examined ambulatory status over time after revascularization and predictors of ambulation loss in CLTI patients.

Methods: We used a clinical database established by the Surgical reconstruction versus Peripheral INtervention in pAtients with critical limb isCHemia study, a prospective, multicentre, observational study including patients with CLTI. The primary endpoint was mobility over time.

Results: Of the 381 patients, the ambulatory proportion at baseline was $71 \%$. The proportion gradually decreased, finally reaching $40 \%$ at 36 months. In non-ambulatory patients at revasacularisation, approximately $20-40 \%$ of patients achieved ambulation. Multivariate analysis confirmed that age, impaired mobility before CLTI onset and at revascularization, renal failure on dialysis, and WIfI clinical stage 4 were positively associated with ambulation loss at either specific or all time points, whereas male sex and surgical reconstruction were inversely associated with the outcomes at specific time points.

Conclusion: Mobility in the overall population gradually decreased, whereas the number of deceased patients increased. Advanced age, impaired mobility before CLTI onset and at revascularization, renal failure on dialysis, and WIfI stage 4 were associated with ambulation loss at almost all points after revascularization.

Clinical trial registration: UMIN000007050.

Key words: Chronic limb-threatening ischemia, Revascularization, Ambulatory, Functional status, Ambulatory status

\section{Introduction}

Chronic limb-threatening ischemia (CLTI) is the most severe form of peripheral arterial disease (PAD) and is characterised by ischaemic rest pain and tissue loss ${ }^{1)}$. The CLTI population is generally frail and has impaired activities of daily living (ADL) and limited lower limb functionality due to ongoing ischaemic

Address for correspondence: Akio Kodama, Division of Vascular Surgery, Department of Surgery, Nagoya University Graduate School of Medicine, 65 Tsurumaicho, Showa-ku, Nagoya 466-8550, Japan E-mail: akodama@med.nagoya-u.ac.jp; kodakio131@gmail.com

Received: March 1, $2021 \quad$ Accepted for publication: April 8, 2021

Copyright@2021 Japan Atherosclerosis Society

This article is distributed under the terms of the latest version of CC BY-NC-SA defined by the Creative Commons Attribution License. 
pain and wounds. Revascularization is needed to improve ischaemic symptoms and avoid major amputation ${ }^{1)}$. The functional status of CLTI patients decreases after major amputation; therefore, one of the major goals in treating CLTI patients is to salvage ischaemic limbs and preserve baseline functional status $^{1,2)}$. Moreover, CLTI is significantly associated with high mid- and long-term mortality ${ }^{1)}$. Therefore, we should consider not only a patient's functional status but also their life expectancy in decision-making regarding appropriate treatment ${ }^{3,4)}$.

Many previous studies have evaluated and used ambulation capability as a surrogate for functional status. Preoperative ambulatory status has been reported to be a predictor of major clinical outcomes, including amputation-free survival and mortality ${ }^{3,5}$. Although several studies have attempted to evaluate patient functional outcomes after revascularization, many of them were single-centre studies, investigated a small number of CLTI patients, and were limited by their relatively restricted scope in assessing functional outcomes because of their retrospective nature ${ }^{6}$. Therefore, the impact of revascularization on ambulatory status remains poorly understood in actual clinical settings.

Although previous studies have revealed several predictors of the ambulatory status and functional outcomes of patients following revascularization, such as amputation and preoperative non-ambulatory status, only a few studies have investigated the factors related to affected limb severity and investigated surviving patients ${ }^{7,8)}$.

\section{Aim}

The aim of the current study was to evaluate ambulatory status over time after revascularization for CLTI and to investigate its associated factors, including not only patient characteristics but also ischaemic limb severity, at each time point in CLTI patients who underwent surgical and endovascular revascularization.

\section{Methods}

We extracted data from a clinical database established by the Surgical reconstruction versus Peripheral INtervention in pAtients with critical limb is CHemia (SPINACH) study, a prospective, multicentre, observational study that registered patients who had CLTI due to atherosclerotic arterial disease in 23 centres (12 vascular surgery departments and 11 interventional cardiology departments) in Japan. The details of the SPINACH study are described elsewhere ${ }^{9,10)}$. The study was performed in accordance with the Declaration of Helsinki and was approved by the ethics committee of each centres. Written informed consent was obtained. The current study analysed 381 CLTI patients who underwent revascularization due to either ischaemic rest pain with Wound, Ischemia, and foot Infection [WIfI] grade 3 ischemia or tissue loss with WIfI grade $2 / 3$ ischemia. Patients who were non-ambulatory before the current CLTI onset with a long-term wheelchair requirement or bedridden status, for example, because of neurogenic deficits (paraplegia, stroke, and so on), who had already undergone major amputation at registration, and who had been diagnosed with dementia, were excluded from the current analysis.

\section{Definitions}

As mentioned previously, the WIfI classes of the study participants were retrospectively determined using photographs of pedal wounds and medical records, including laboratory examinations at the time of registration ${ }^{11)}$. The initial judgement was made at each participating centre and was subsequently reviewed by an independent plastic surgeon. Disagreements were discussed and resolved in a subsequent committee meeting attended by a plastic surgeon, a vascular surgeon and an interventional cardiologist. Skin perfusion pressures (SPPs) of 31-40 $\mathrm{mmHg}$ and $\leq 30 \mathrm{mmHg}$ were considered WIfI grade 2 and 3 ischemia, respectively. The ambulatory status immediately before revascularization for the current CLTI and at each time point after revascularization was classified into the following categories: (1) ambulatory without aid (i.e., independently ambulatory), (2) ambulatory with aid (i.e., ambulatory using crutches/cane/walker), (3) wheelchair without aid (i.e., operates a wheelchair without aid), (4) wheelchair with aid (i.e., operates a wheel chair assisted by another person), (5) bedridden, and (6) deceased. The data on ambulatory status before the current CLTI onset were obtained from medical records or self-reports. Renal failure was defined as an estimated glomerular filtration rate $<30 \mathrm{ml} / \mathrm{min} / 1.73$ $\mathrm{m}^{2}$ or dependence on dialysis. During the three-year follow-up period, ambulatory status was scheduled to be assessed at 3, 12, 24, and 36 months, with a tolerated deviation of \pm 1 month.

\section{Outcome Measures}

The outcome measure in the current study was ambulation, i.e., survival with ambulation regardless of aid. Ambulation loss indicated a wheelchair-bound, bedridden, or deceased status. 
Table 1. Characteristics of the study population

\begin{tabular}{lc}
\hline$N$ & 381 \\
\hline Age (years) & $72 \pm 9$ \\
Male sex & $266(70 \%)$ \\
Ambulation with aid before CLTI onset & $89(23 \%)$ \\
Mobility at revascularization & \\
$\quad$ Ambulatory without aid & $193(51 \%)$ \\
$\quad$ Ambulatory with aid & $77(20 \%)$ \\
$\quad$ In wheelchair without aid & $69(18 \%)$ \\
$\quad$ In wheelchair with aid & $38(10 \%)$ \\
$\quad$ Bedridden & $4(1 \%)$ \\
Current smoking & $64(17 \%)$ \\
Diabetes mellitus & $285(75 \%)$ \\
Renal failure & \\
$\quad$ None (eGFR $\geq 30$ ml/min/1.73 m $\left.^{2}\right)$ & $160(42 \%)$ \\
$\quad$ Renal failure without dialysis & $19(5 \%)$ \\
$\quad$ Renal failure on dialysis & $202(53 \%)$ \\
Heart failure & $64(17 \%)$ \\
Haemoglobin (g/dl) & $11.1 \pm 1.9$ \\
WIfl clinical stage & \\
$\quad$ Stage 2 & $69(18 \%)$ \\
Stage 3 & $117(31 \%)$ \\
Stage 4 & $195(51 \%)$ \\
Surgical reconstruction & $372(98 \%)$ \\
\hline
\end{tabular}

\section{Statistical Analysis}

Data are presented as the mean and standard deviation (SD) for continuous variables or as percentages for discrete variables, if not otherwise mentioned. A $P$ value of $<.05$ was considered statistically significant, and $95 \%$ confidence intervals are reported when appropriate. The proportions of the outcome measures at specific time points were compared with those at baseline using the McNemar test. The associations of baseline characteristics with either ambulation loss or independent ambulation loss were investigated with binomial logistic regression models. We also supplementarily investigated the association between limb status and ambulation at each time point. Missing data were treated with the use of multiple imputation (10 times) by the chained equations method. All statistical analyses were performed using $\mathrm{R}$ version 3.6.0 ( $\mathrm{R}$ Development Core Team, Vienna, Austria).

\section{Results}

The baseline characteristics of the study population are summarized in Table 1 . The mean age was $72 \pm 9$ years, and the prevalence of diabetes mellitus and renal failure was $75 \%$ and $58 \%$, respectively. WIfI clinical stage $1,2,3$, and 4 accounted for $0 \%, 18 \%, 31 \%$, and $51 \%$, respectively. Forty percent of the population underwent surgical reconstruction, and the rest underwent only endovascular therapy. In the patients undergoing endovascular treatment (EVT) alone, 102 patients (44\%) underwent stent implantation (19 patients [8\%] drug-eluting stent implantation), and 167 (73\% of only endovascular therapy patients) underwent infrapopliteal revascularization. In the patients undergoing surgical reconstruction, distal anastomoses were performed on crural or pedal arteries in 113 patients ( $75 \%$ of surgical reconstruction patients).

Fig. 1 illustrates the changes in mobility during the three years after revascularization. The estimated proportion of ambulation in the overall population [95\% confidence interval] was $71 \%$ [66\% to $75 \%]$ at baseline, and thereafter gradually decreased $(P<.05$ versus baseline), finally reaching $40 \%$ [35\% to $45 \%$ ] at 36 months. The estimated mortality rates at 12,24 , 36 months were $17 \%$ [ $13 \%$ to $20 \%$ ], $29 \%$ [ $24 \%$ to $33 \%$ ], and 43\% [38\% to 48\%], respectively (Fig. 1A). On the other hand, in patients without ambulation at revascularization, approximately $40 \%$ of patients achieved ambulation after revascularization, and the proportion of patients achieving ambulation was

\section{Advance Publication Journal of Atherosclerosis and Thrombosis}



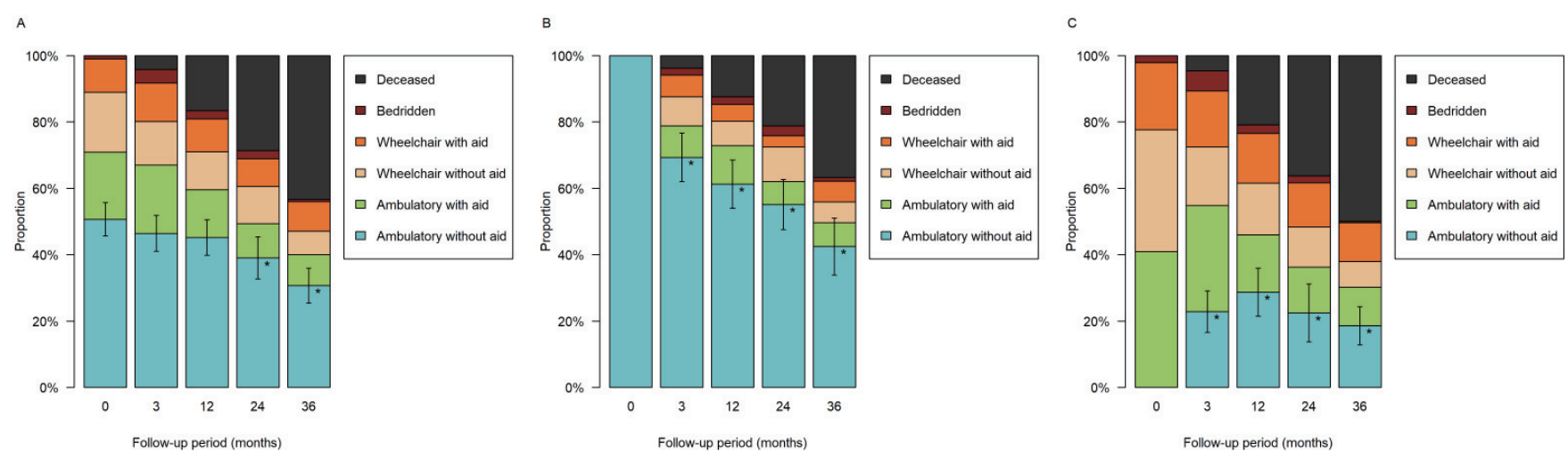

Fig. 1. Change in mobility status

Panels A to C illustrate the proportions of respective mobility statuses in the overall population (A), in patients with non-ambulation (i.e., in a wheelchair regardless of aid or bedridden) at baseline (B), and in patients who were ambulatory regardless of aid at baseline (C). Error bars indicate $95 \%$ confidence intervals for the proportion of patients who were ambulatory regardless of aid, and asterisks represent $P<.05$ versus the baseline value regarding the proportion of patients who were ambulatory regardless of aid.

significantly increased throughout the three-year follow-up (Fig. 1B). However, it was much lower than that in those who were ambulatory at baseline (Fig. 1C). Similar trends were observed when the outcome measure was independent ambulation instead of ambulation regardless of aid (Supplementary Fig. 1A to C).

The associations of baseline characteristics with ambulation loss and independent ambulation loss during the follow-up period are shown in Table 2 and Supplementary Table 1. Age, impaired mobility before CLTI onset and at revascularization, renal failure on dialysis, and WIfI clinical stage 4 were positively associated with the outcomes at either specific or all time points, whereas male sex and surgical reconstruction were inversely associated with the outcomes at specific time points. There was no significant interaction effect of non-ambulatory status at revascularization on the associations of baseline characteristics with independent ambulation loss or ambulation loss (all $P>.05$ ) (data not shown).

In patients who were not ambulatory at revascularization $(29 \%$ of the total cohort), ambulation with versus without aid before CLTI onset, renal failure on dialysis, and WIfI clinical stage 4 were associated with ambulation loss during the follow-up period (Supplementary Table 2). In other words, among patients who were not ambulatory at revascularization, those who had been ambulatory without aid before CLTI onset, those were not on dialysis, and those who were free from WIfI clinical stage 4 were likely to restore the walking ability after revascularization. However, it should be noted that the sample size of this sub-analysis was so small (111 patients were included and the outcomes were observed only in 20 40\%) that the multivariate regression models might be subject to overfitting. Future studies with a larger number of this subgroup population will be needed to validate the current findings.

The association of limb status after revascularizasion and ambulation loss were also investigated (Supplementary Fig. 2 and Supplementary Table 3). Patients with major amputation and those with tissue loss were more likely to have ambulation loss ().

\section{Discussion}

In this study, we demonstrated short- and midterm changes in mobility after revascularization, which were stratified by ambulation status at revascularization. Moreover, we revealed some predictors of ambulation loss, including not only patient characteristics but also affected limb severity, by using the WIfI stage. In the current study, we applied only preoperative variables in the logistic regression model to clarify the risk stratification of ambulation loss after revascularization in actual clinical settings. With respect to mobility over time in the overall cohort, it gradually decreased until 3 years, and the proportion of deceased patients increased. Approximately $20-40 \%$ of patients without ambulation at revascularization recovered their functional capacity after revascularization until 3 years. Multivariate analysis confirmed that renal failure on dialysis was associated with ambulatory loss after revascularization throughout the study period. WIfI clinical stage 4 was also associated with this outcome in the short-term (within a year) after 
Table 2. Associations of baseline characteristics with ambulation loss

\begin{tabular}{|c|c|c|}
\hline & Crude odds ratio & Adjusted odds ratio \\
\hline \multicolumn{3}{|l|}{ Age (per 10 years) } \\
\hline At 3 months & $1.3[1.0$ to 1.7$](P=.029)^{*}$ & $1.5[1.1$ to 2.0$](P=.010)^{*}$ \\
\hline At 12 months & $1.3[1.0$ to 1.6$](P=.084)$ & $1.4[1.0$ to 2.0$](P=.086)$ \\
\hline At 24 months & $1.3[1.0$ to 1.7$](P=.028)^{*}$ & $1.4[1.0$ to 1.9$](P=.026)^{*}$ \\
\hline At 36 months & $1.0[0.8$ to 1.3$](P=.88)$ & $1.1[0.8$ to 1.4$](P=.60)$ \\
\hline \multicolumn{3}{|c|}{ Male sex (vs. female sex) } \\
\hline At 3 months & $0.6[0.4$ to 1.0$](P=.044)^{*}$ & $0.7[0.4$ to 1.2$](P=.21)$ \\
\hline At 12 months & $0.5[0.3$ to 0.9$](P=.010)^{*}$ & $0.5[0.3$ to 1.0$](P=.056)$ \\
\hline At 24 months & $0.8[0.5$ to 1.3$](P=.44)$ & $1.1[0.6$ to 2.0$](P=.71)$ \\
\hline At 36 months & $1.2[0.8$ to 2.0$](P=.39)$ & $1.5[0.8$ to 2.7$](P=.23)$ \\
\hline \multicolumn{3}{|c|}{ Ambulatory with aid before CLI onset (vs. ambulatory without aid) } \\
\hline At 3 months & $2.2[1.3$ to 3.6$](P=.002)^{*}$ & $2.1[0.9$ to 4.6$](P=.068)$ \\
\hline At 12 months & $3.1[1.8$ to 5.3$](P<.001)^{*}$ & $3.1[1.3$ to 7.1$](P=.009)^{*}$ \\
\hline At 24 months & $3.1[1.7$ to 5.5$](P<.001)^{*}$ & $2.9[1.1$ to 7.2$](P=.025)^{*}$ \\
\hline At 36 months & $2.1[1.0$ to 4.2$](P=.040)^{*}$ & $2.2[0.9$ to 5.3$](P=.084)$ \\
\hline \multicolumn{3}{|c|}{ Ambulatory with aid at revascularization (vs. ambulatory without aid) } \\
\hline At 3 months & $1.2[0.6$ to 2.3$](P=.65)$ & $0.6[0.2$ to 1.6$](P=.34)$ \\
\hline At 12 months & $1.8[1.0$ to 3.3$](P=.045)^{*}$ & $0.8[0.3$ to 1.9$](P=.61)$ \\
\hline At 24 months & $1.8[1.0$ to 3.2$](P=.048)^{*}$ & $0.9[0.4$ to 2.2$](P=.90)$ \\
\hline At 36 months & $1.3[0.7$ to 2.6$](P=.43)$ & $0.9[0.4$ to 2.1$](P=.82)$ \\
\hline \multicolumn{3}{|c|}{ Non-ambulatory at revascularization (vs. ambulatory without aid) } \\
\hline At 3 months & $5.6[3.1$ to 10.2$](P<.001)^{*}$ & $3.4[1.6$ to 7.2$](P=.001)^{*}$ \\
\hline At 12 months & $4.7[2.8$ to 7.8$](P<.001)^{*}$ & $2.7[1.4$ to 5.2$](P=.002)^{*}$ \\
\hline At 24 months & $4.2[2.3$ to 7.4$](P<.001)^{*}$ & $2.7[1.3$ to 5.6$](P=.011)^{*}$ \\
\hline At 36 months & $3.6[1.7$ to 7.7$](P=.002)^{*}$ & $2.6[1.0$ to 6.9$](P=.051)$ \\
\hline \multicolumn{3}{|l|}{ Current smoking } \\
\hline At 3 months & $0.4[0.2$ to 0.8$](P=.009)^{*}$ & $0.8[0.3$ to 1.7$](P=.50)$ \\
\hline At 12 months & $0.4[0.2$ to 0.9$](P=.022)^{*}$ & $0.7[0.3$ to 1.7$](P=.45)$ \\
\hline At 24 months & $0.5[0.2$ to 0.9$](P=.025)^{*}$ & $0.7[0.3$ to 1.7$](P=.46)$ \\
\hline At 36 months & $0.7[0.4$ to 1.2$](P=.21)$ & $1.0[0.5$ to 2.0$](P=.96)$ \\
\hline \multicolumn{3}{|l|}{ Diabetes mellitus } \\
\hline At 3 months & $1.5[0.9$ to 2.7$](P=.15)$ & $1.3[0.6$ to 2.6$](P=.48)$ \\
\hline At 12 months & $1.2[0.7$ to 2.0$](P=.47)$ & $0.9[0.5$ to 1.7$](P=.82)$ \\
\hline At 24 months & $1.4[0.9$ to 2.3$](P=.15)$ & $1.2[0.7$ to 2.1$](P=.45)$ \\
\hline At 36 months & $1.7[1.1$ to 2.8$](P=.029)^{*}$ & $1.4[0.8$ to 2.4$](P=.23)$ \\
\hline \multicolumn{3}{|c|}{ Renal failure without dialysis (vs eGFR $\geq 30 \mathrm{ml} / \mathrm{min} / 1.73 \mathrm{~m}^{2}$ ) } \\
\hline At 3 months & $0.6[0.1$ to 2.7$](P=.52)$ & $0.8[0.2$ to 3.9$](P=.82)$ \\
\hline At 12 months & $1.6[0.5$ to 4.8$](P=.41)$ & $2.3[0.7$ to 7.9$](P=.19)$ \\
\hline At 24 months & $1.0[0.4$ to 2.9$](P=.95)$ & $0.9[0.3$ to 2.7$](P=.81)$ \\
\hline At 36 months & $1.2[0.4$ to 3.3$](P=.72)$ & $1.0[0.3$ to 3.0$](P=.98)$ \\
\hline \multicolumn{3}{|c|}{ Renal failure on dialysis (vs eGFR $\geq 30 \mathrm{ml} / \mathrm{min} / 1.73 \mathrm{~m}^{2}$ ) } \\
\hline At 3 months & $2.5[1.6$ to 4.2$](P<.001)^{*}$ & $3.0[1.7$ to 5.4$](P<.001)^{*}$ \\
\hline At 12 months & $3.2[1.9$ to 5.3$](P<.001)^{*}$ & $4.6[2.2$ to 9.9$](P<.001)^{*}$ \\
\hline At 24 months & $3.1[1.9$ to 5.1$](P<.001)^{*}$ & $3.3[1.8$ to 6.1$](P<.001)^{*}$ \\
\hline At 36 months & $3.3[1.9$ to 5.7$](P<.001)^{*}$ & $3.0[1.6$ to 5.6$](P=.001)^{*}$ \\
\hline
\end{tabular}




\begin{tabular}{|c|c|c|}
\hline & Crude odds ratio & Adjusted odds ratio \\
\hline \multicolumn{3}{|l|}{ Heart failure } \\
\hline At 3 months & $1.5[0.8$ to 2.8$](P=.21)$ & $1.0[0.5$ to 2.2$](P=.90)$ \\
\hline At 12 months & $1.5[0.8$ to 2.7$](P=.19)$ & $1.0[0.5$ to 2.1$](P=.96)$ \\
\hline At 24 months & $1.6[0.8$ to 3.2$](P=.17)$ & $1.0[0.5$ to 2.4$](P=.91)$ \\
\hline At 36 months & $1.9[1.0$ to 3.7$](P=.048)^{*}$ & $1.4[0.7$ to 2.9$](P=.39)$ \\
\hline \multicolumn{3}{|c|}{ Haemoglobin (per $1 \mathrm{~g} / \mathrm{dl}$ ) } \\
\hline At 3 months & $0.9[0.8$ to 1.0$](P=.026)^{*}$ & $1.0[0.9$ to 1.2$](P=.81)$ \\
\hline At 12 months & $0.9[0.8$ to 1.0$](P=.034)^{*}$ & $1.1[0.9$ to 1.3$](P=.30)$ \\
\hline At 24 months & $0.8[0.7$ to 0.9$](P=.002)^{*}$ & $1.0[0.8$ to 1.1$](P=.52)$ \\
\hline At 36 months & $0.8[0.7$ to 0.9$](P=.006)^{*}$ & $1.0[0.8$ to 1.1$](P=.56)$ \\
\hline \multicolumn{3}{|c|}{ WIfI clinical stage 4 (vs. WIfI clinical stage $2 / 3$ ) } \\
\hline At 3 months & $2.0[1.3$ to 3.1$](P=.003)^{*}$ & $2.1[1.2$ to 3.7$](P=.010)^{*}$ \\
\hline At 12 months & $2.1[1.4$ to 3.2$](P=.001)^{*}$ & $2.9[1.6$ to 5.0$](P<.001)^{*}$ \\
\hline At 24 months & $1.4[0.9$ to 2.2$](P=.15)$ & $1.6[0.9$ to 2.8$](P=.085)$ \\
\hline At 36 months & $1.3[0.8$ to 2.1$](P=.21)$ & $1.2[0.7$ to 1.9$](P=.54)$ \\
\hline \multicolumn{3}{|c|}{ Surgical reconstruction (vs. EVT alone) } \\
\hline At 3 months & $0.9[0.6$ to 1.4$](P=.65)$ & $0.9[0.5$ to 1.5$](P=.59)$ \\
\hline At 12 months & $0.7[0.4$ to 1.0$](P=.067)$ & $0.6[0.3$ to 1.0$](P=.038)^{*}$ \\
\hline At 24 months & $0.5[0.3$ to 0.8$](P=.007)^{*}$ & $0.5[0.3$ to 0.8$](P=.006)^{*}$ \\
\hline At 36 months & $0.8[0.5$ to 1.4$](P=.42)$ & $0.8[0.5$ to 1.4$](P=.46)$ \\
\hline
\end{tabular}

Data are odds ratios for ambulation loss (regardless of aid) at 3,12, 24, and 36 months [95\% confidence intervals] $(P$ values). Crude odds ratios were derived from the univariate logistic regression model, whereas adjusted odds ratios were derived from the multivariate logistic regression model in which all the variables listed in the tables were entered as explanatory variables. Asterisks indicate $P<.05$.

revascularization.

In clinical decision-making, to preserve functional status after revascularization, physicians should consider life expectancy because the CLTI population has a generally poor survival rate. Although multiple risk stratification tools to estimate life expectancy have been developed and applied to the CLTI population, the recently published guidelines state that no specific model can be recommended ${ }^{4)}$. Indeed, we cannot predict mortality in each patient in an actual clinical setting. Therefore, we analysed ambulatory status over time in all CLTI patients, which included not only surviving but also deceased patients ${ }^{9}$. This was a deliberate choice by the participants of the SPINACH study to combine mortality and ambulatory status, which is different from other analyses. Obviously this influences the outcome rates which should be kept in mind. Several studies reported that the rates of maintenance of ambulation at 1 and 3 years were $70-90 \%$ and $70-80 \%$, respectively ${ }^{7,12}$. These outcomes seemed to be much better than our results. However, these studies retrospectively assessed only surviving patients at each time point; therefore, the proportion of ambulatory patients was higher than that in studies including deceased patients. Our results with regard to the proportion of ambulation after revascularization were comparable to some previous reports, which analysed overall patients, including deceased patients ${ }^{13,14)}$.

Very few multicentre prospective studies have evaluated mobility over time after revascularization in CLTI cohorts ${ }^{8,15)}$. Goodney et al. examined 1400 patients who underwent surgical operation, of whom CLTI patients accounted for $75 \%$. They reported that 93\% of CLTI patients were ambulatory preoperatively, and $75 \%$ remained ambulatory at 1 year. This study demonstrated novel findings by showing the proportion of deceased patients, but the follow-up period was short (1 year) and did not include the endovascular treatment (EVT) cohort. Duffy et al. evaluated procedural and functional outcomes in 1864 CLTI patients and focused on differences between sexes. Notably, they found that both females and males had a deteriorated ambulatory status at discharge but an improved status at 1 year, and females were more likely to be non-ambulatory than males. However, they also evaluated ambulatory status in only surviving patients. Furthermore, they did not investigate the predictors of functional capacity 
maintenance with preoperative measurements. In contrast, the current study revealed mobility over time after both EVT and surgical procedures during the mid-term period and tried to identify predictors considering preoperative variables, including limb severity, in real-world settings. A recently published meta-analysis of the natural history of untreated CLTI reported that both all-cause mortality and major amputation rate at 12 months were $22 \%{ }^{16}$ ). Several studies demonstrated that major amputation has been reported to be associated with reduced mobility ${ }^{8,17)}$. Furthermore, despite initial successful prosthetic rehabilitation, prosthetic use deteriorates over time ${ }^{18)}$. Indeed, our results did not show promise in terms of mobility after revascularization at mid-term follow-up, but the impact of ravascularisation on mobility in CLTI patients needs to be clarified in the near future.

There are multiple factors that contribute to functional status, including age, severity of comorbidities, physiologic reserves, severity of affected limb, dementia, and so on. In short, functional capacity is significantly affected by patient and limb backgrounds. Therefore, it is difficult to select an appropriate treatment on the basis of preoperative risk factors for ambulatory loss. Several studies have reported that preoperative impaired mobility negatively affects the improvement of functional outcomes $^{8,}$ 13, 19). Vogel et al. reported that poor baseline mobility was significantly associated with a worsening functional status over time ${ }^{19)}$. Flu et al. reported that non-ambulatory patients had an increase in the occurrence of adverse events and poor longterm survival rates and did not receive a benefit from revascularization after six years in terms of functional status improvement ${ }^{19)}$. The study in poor-risk CLTI patients who required assistance for daily life by lida $e t$ al. showed no improvement in ADL scores after revascularization ${ }^{20)}$. The current study also revealed that non-ambulatory status at revascularization was one of the independent predictors of ambulatory loss. However, approximately $30-50 \%$ of patients with a preprocedural non-ambulatory status achieved improved mobility within 6 months after revascularization. We should distinguish these patients despite their impaired mobility at revascularization in clinical decision-making.

We found that renal failure on dialysis was also associated with ambulation loss at almost all time points after revascularization. One possible explanation is that patients with renal failure on dialysis were unlikely to recover even after revascularization because renal failure on dialysis has been associated with frailty in several studies ${ }^{21)}$. Another possible explanation is the high mortality in
CLTI patients with renal failure on dialysis ${ }^{5)}$. The current analysis included deceased patients at each time point after revascularization. Indeed, renal failure on dialysis was one variable in the predictive model of mortality derived from the SPINACH cohort $^{4}$.

The presence of ischaemic lesions at presentation was shown to affect short-term functionality and postoperative morbidity in a previous univariate analysis ${ }^{22}$. However, whether the severity of pedal necrosis or infection affects functional outcome after revascularization is unknown. The WIfI classification system has been reported to be associated with limb prognosis, including wound healing of ischaemic ulcer prognoses $^{23)}$. We used the WIfI clinical stage as a surrogate for the severity of ischaemic wounds. Hata et al reported WIfI stage 4 as one of the independent predictors of wound healing after $\mathrm{EVT}^{24)}$. A possible explanation would be delayed rehabilitation due to severe ischaemic wounds and prolonged wound healing time. These factors might contribute to the need for long hospitalization times. Hospitalization itself has been reported to be associated with substantial and sustained declines in $\mathrm{ADL}^{19}$.

In the current study, surgical reconstruction was inversely associated with (independent) ambulation loss during the short term after revascularization. Vogel et al. demonstrated that EVT was associated with a less favourable functional status than after surgical reconstruction ${ }^{19)}$. This result might be explained by the fact that, in the SPINACH cohort, the wound healing rates at 1,3 , and 6 months after surgical reconstruction were better than those after EVT in the secondary propensity score matched population, as was previously published by our group $^{11)}$. Furthermore, several studies have shown superior wound healing with bypass surgery ${ }^{25)}$. Indeed, tissue loss at 3, 12, and 36 months after revascularization was associated with ambulation loss (Supplementary Table 2). Another possible explanation for this is that there might be a selection bias when the treatment strategy was determined by a team of vascular specialists. In general, surgical reconstruction is more invasive than angioplasty. Therefore, patients who underwent surgical treatment might be less frail than patients who underwent angioplasty. However, the cause-and-effect relationship (whether bypass surgery reduces the risk of ambulation loss) is unknown in the present study. An intervention trial is needed to clarify whether surgical treatment could reduce the risk of ambulation loss. The BESTCLI, BASIL-2, and BASIL-3 studies could show the superiority between angioplasty and bypass surgery in terms of functional status ${ }^{26-28)}$.

This study has several limitations. First, this 
study was an observational study; therefore, revascularization strategies were chosen by clinicians, and hence, some relevant bias may be present. Second, the WIfI classification of each patient could be a potential bias, although data were prospectively collected and reviewed by an independent plastic surgeon. Furthermore, the ischemic grade was determined based on the SPP value especially when assessing ischemic limbs with severely calcified noncompressible arteries. The boundary values of SPP for the WIfI ischaemic grade have not been clarified at the moment, therefore, it could also be a potential bias. Third, the rehabilitation programme was different at each institution. Rehabilitation after revascularization has a strong impact on the recovery of functional status ${ }^{29)}$. CLTI cohorts are generally frail and need rehabilitation to maintain mobility. Fourth, the causes of ambulation loss related to ambulatory loss, including the postoperative factors such as ischaemic pain status were not clarified. These factors might influence ambulatory status. Finally, this study did not analyse cause and effect relationship. Although this study has several limitations, we believe that it offers useful information to clinicians regarding clinical decision-making for the management of CLTI patients.

\section{Conclusion}

In the current study, we investigated mobility over time in CLTI patients who underwent revascularization and identified some predictors of ambulation loss. The estimated proportion of ambulation in the overall population gradually decreased, whereas the number of deceased patients increased. However, in patients without ambulation at revascularization, approximately $20-40 \%$ of patients achieved ambulation during 36 months after revascularization. Advanced age, impaired mobility before CLTI onset and at revascularization, renal failure on dialysis, and WIfI stage 4 were strongly associated with ambulation loss during the follow-up period. Consideration of patient and limb characteristics and preoperative functional status are necessary for clinicians to decide a treatment strategy considering patient-oriented outcomes. Further studies are needed to clarify mobility over time after revascularization and the differences between EVT and surgical bypass with uniform rehabilitation programmes and wound management with multicentre, prospective, randomized control trials.

\section{Sources of Funding}

The SPINACH study (Surgical Reconstruction Versus Peripheral Intervention in Patients With Critical Limb Ischemia) was sponsored by Abbott Vascular Japan Co, Ltd, Boston Scientific Japan K.K., Cook Japan Incorporated, Goodman Co, Ltd, Johnson \& Johnson K.K., Kaken Pharmaceutical Co, Ltd, Kaneka Medix Corporation, Medicon Inc, Medikit Co, Ltd, Medtronic Japan Co, Ltd, Mitsubishi Tanabe Pharma Corporation, MSD K.K., St. Jude Medical Japan Co, Ltd, Taisho Toyama Pharmaceutical Co, Ltd, Terumo Corp, and W.L. Gore \& Associates, Co, Ltd (in alphabetic order).

\section{Conflicts of Interest}

There are no conflicts of interests associated with this manuscript.

\section{References}

1) Norgren L, Hiatt WR, Dormandy JA, Nehler MR, Harris $\mathrm{KA}$, Fowkes FG. Inter-Society Consensus for the Management of Peripheral Arterial Disease (TASC II). J Vasc Surg, 2007; 45 Suppl S: S5-67

2) Luther M. Surgical Treatment of Chronic Critical Leg Ischaemia: A Five-Year Follow-Up of Survival, Mobility, and Treatment Level. European Journal of Surgery, 1998; 164: 35

3) Simons JP, Schanzer A, Flahive JM, Osborne NH, Mills JL, Sr., Bradbury AW, Conte MS. Survival prediction in patients with chronic limb-threatening ischemia who undergo infrainguinal revascularization. J Vasc Surg, 2019; 69: 137S-151S e133

4) Azuma N, Takahara M, Kodama A, Soga Y, Terashi H, Tazaki J, Yamaoka T, Koya A, Iida O. Predictive Model for Mortality Risk Including the Wound, Ischemia, Foot Infection Classification in Patients Undergoing Revascularization for Critical Limb Ischemia. Circ Cardiovasc Interv, 2019; 12: e008015

5) Kodama A, Sugimoto M, Kuma S, Okazaki J, Mii S, Komori K. Clinical outcomes after infrainguinal bypass grafting for critical limb ischaemia in patients with dialysis-dependent end-stage renal failure. Eur J Vasc Endovasc Surg, 2014; 48: 695-702

6) Rollins KE, Coughlin PA. Functional outcomes following revascularisation for critical limb ischaemia. Eur J Vasc Endovasc Surg, 2012; 43: 420-425

7) Taylor SM, Kalbaugh CA, Blackhurst DW, Cass AL, Trent EA, Langan EM, 3rd, Youkey JR. Determinants of functional outcome after revascularization for critical limb ischemia: an analysis of 1000 consecutive vascular interventions. J Vasc Surg, 2006; 44: 747-755; discussion 755-746

8) Goodney PP, Likosky DS, Cronenwett JL, Vascular Study Group of Northern New E. Predicting ambulation status one year after lower extremity bypass. J Vasc Surg, 2009; 
49: 1431-1439 e1431

9) Azuma N, Iida O, Takahara M, Soga Y, Kodama A. Surgical reconstruction versus peripheral intervention in patients with critical limb ischemia - a prospective multicenter registry in Japan: the SPINACH study design and rationale. Vascular, 2014; 22: 411-420

10) Takahara M, Iida O, Soga Y, Kodama A, Terashi H, Azuma N, SPINACH study investigators. Current and Past Obesity in Japanese Patients with Critical Limb Ischemia Undergoing Revascularization. J Atheroscler Thromb, 2021; 28: 44-51

11) Iida O, Takahara M, Soga Y, Kodama A, Terashi H, Azuma N, SPINACH study investigators. Three-Year Outcomes of Surgical Versus Endovascular Revascularization for Critical Limb Ischemia: The SPINACH Study (Surgical Reconstruction Versus Peripheral Intervention in Patients With Critical Limb Ischemia). Circ Cardiovasc Interv, 2017; 10

12) Chung J, Bartelson BB, Hiatt WR, Peyton BD, McLafferty RB, Hopley CW, Salter KD, Nehler MR. Wound healing and functional outcomes after infrainguinal bypass with reversed saphenous vein for critical limb ischemia. J Vasc Surg, 2006; 43: 1183-1190

13) Flu HC, Lardenoye JH, Veen EJ, Van Berge Henegouwen DP, Hamming JF. Functional status as a prognostic factor for primary revascularization for critical limb ischemia. J Vasc Surg, 2010; 51: 360-371 e361

14) Kumar BN, Gambhir RP. Critical limb ischemia-need to look beyond limb salvage. Ann Vasc Surg, 2011; 25: 873877

15) Duffy RP, Adams JE, Callas PW, Schanzer A, Goodney PP, Ricci MA, Cronenwett JL, Bertges DJ, Vascular Study Group of New England. The influence of gender on functional outcomes of lower extremity bypass. J Vasc Surg, 2014; 60: 1282-1290 e1281

16) Abu Dabrh AM, Steffen MW, Undavalli C, Asi N, Wang $\mathrm{Z}$, Elamin MB, Conte MS, Murad MH. The natural history of untreated severe or critical limb ischemia. J Vasc Surg, 2015; 62: 1642-1651 e1643

17) Deneuville M, Perrouillet A. Survival and quality of life after arterial revascularization or major amputation for critical leg ischemia in Guadeloupe. Ann Vasc Surg, 2006; 20: 753-760

18) Conte MS, Bradbury AW, Kolh P, White JV, Dick F, Fitridge R, Gvg Writing Group for the Joint Guidelines of the Society for Vascular Surgery, European Society for Vascular Surgery World Federation of Vascular, Societies Global Vascular Guidelines on the Management of Chronic Limb-Threatening Ischemia. Eur J Vasc Endovasc Surg, 2019; 58: S1-S109 e133

19) Vogel TR, Petroski GF, Kruse RL. Functional status of elderly adults before and after interventions for critical limb ischemia. J Vasc Surg, 2014; 59: 350-358

20) Iida O, Takahara M, Soga Y, Azuma N, Nanto S,
Uematsu M, Priority Investigators. Prognostic Impact of Revascularization in Poor-Risk Patients With Critical Limb Ischemia: The PRIORITY Registry (Poor-Risk Patients With and Without Revascularization Therapy for Critical Limb Ischemia). JACC Cardiovasc Interv, 2017; 10: 1147-1157

21) Chowdhury R, Peel NM, Krosch M, Hubbard RE. Frailty and chronic kidney disease: A systematic review. Arch Gerontol Geriatr, 2017; 68: 135-142

22) Abou-Zamzam AM, Lee RW, Moneta GL, Taylor LM, Porter JM. Functional outcome after infrainguinal bypass for limb salvage. Journal of Vascular Surgery, 1997; 25: 287-297

23) Weaver ML, Hicks CW, Canner JK, Sherman RL, Hines KF, Mathioudakis N, Abularrage CJ. The Society for Vascular Surgery Wound, Ischemia, and foot Infection (WIfI) classification system predicts wound healing better than direct angiosome perfusion in diabetic foot wounds. J Vasc Surg, 2018; 68: 1473-1481

24) Hata $Y$, Iida O, Okamoto $S$, Ishihara $T$, Nanto $K$, Tsujumura T, Takahara M, Mano Y. Additional Risk Stratification Using Local and Systemic Factors for Patients with Critical Limb Ischaemia Undergoing Endovascular Therapy in the WIfI Era. Eur J Vasc Endovasc Surg, 2019; 58: 548-555

25) Spillerova K, Biancari F, Leppaniemi A, Alback A, Soderstrom M, Venermo M. Differential impact of bypass surgery and angioplasty on angiosome-targeted infrapopliteal revascularization. Eur J Vasc Endovasc Surg, 2015; 49: 412-419

26) Menard MT, Farber A. The BEST-CLI trial: a multidisciplinary effort to assess whether surgical or endovascular therapy is better for patients with critical limb ischemia. Semin Vasc Surg, 2014; 27: 82-84

27) Popplewell MA, Davies H, Jarrett H, Bate G, Grant M, Patel S, Mahta S, Andronis L, Roberts T, Deeks J, Bradbury A, Basil Trial Investigators. Bypass versus angio plasty in severe ischaemia of the leg - 2 (BASIL-2) trial: study protocol for a randomised controlled trial. Trials, 2016; 17: 11

28) Hunt BD, Popplewell MA, Davies H, Meecham L, Jarrett H, Bate G, Grant M, Petel S, Hewitt C, Andronis L, Deeks JJ, Bradbury A, Basil Trial Investigators. BAlloon versus Stenting in severe Ischaemia of the Leg-3 (BASIL3): study protocol for a randomised controlled trial. Trials, 2017; 18: 224

29) Gerhard-Herman MD, Gornik HL, Barrett C, Barshes NR, Corriere MA, Drachman DE, et al. 2016 AHA/ACC Guideline on the Management of Patients With Lower Extremity Peripheral Artery Disease: A Report of the American College of Cardiology/American Heart Association Task Force on Clinical Practice Guidelines. Circulation, 2017; 135: e726-e779 

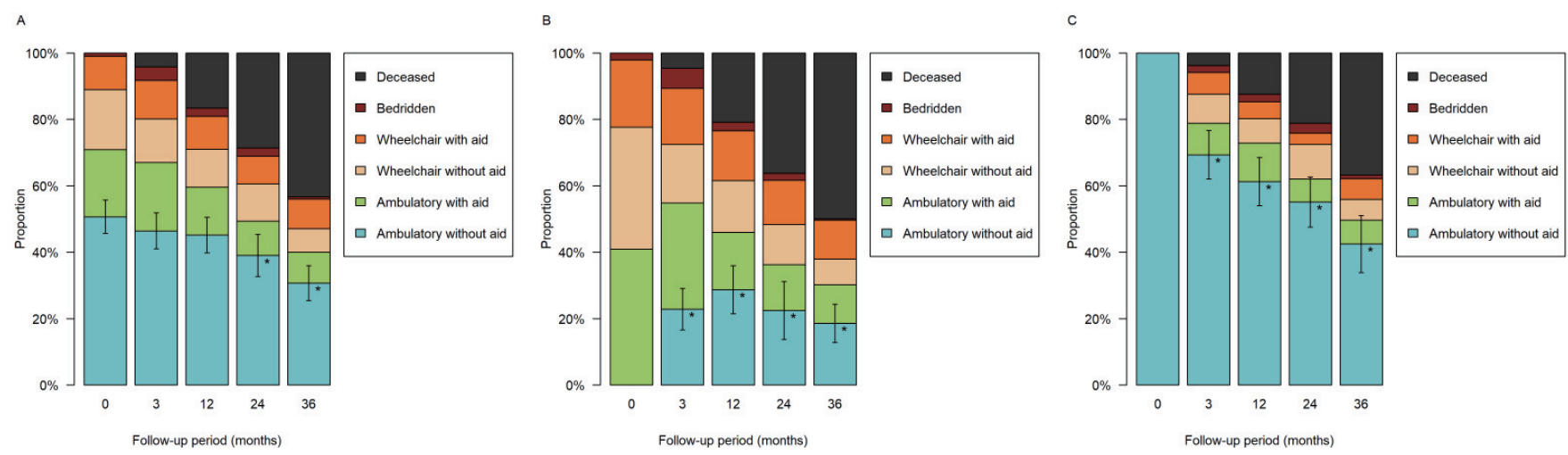

Supplementary Fig. 1. Change in mobility status

Panels A to C illustrate the proportions of the respective mobility statuses in the overall population (A), in a wheelchair regardless of aid, or bedridden) at baseline (B), and in patients who were ambulatory without aid (i.e., independently ambulatory) at baseline (C). Error bars indicate $95 \%$ confidence intervals for the proportion of patients who were ambulatory without aid (i.e., independently ambulatory), and asterisks represent $P<.05$ versus the baseline value regarding the proportion of patients who were ambulatory without aid. 
Supplementary Table 1. Associations of baseline characteristics with independent ambulation loss

\begin{tabular}{|c|c|c|}
\hline & Crude odds ratio & Adjusted odds ratio \\
\hline \multicolumn{3}{|l|}{ Age (per 10 years) } \\
\hline At 3 months & $1.2[1.0$ to 1.6$](P=.051)$ & $1.2[0.9$ to 1.6$](P=.23)$ \\
\hline At 12 months & $1.4[1.0$ to 1.8$](P=.031)^{*}$ & $1.4[1.0$ to 1.9$](P=.055)$ \\
\hline At 24 months & $1.3[1.0$ to 1.6$](P=.064)$ & $1.3[1.0$ to 1.8$](P=.10)$ \\
\hline At 36 months & $1.2[0.8$ to 1.6$](P=.36)$ & $1.2[0.8$ to 1.7$](P=.43)$ \\
\hline \multicolumn{3}{|c|}{ Male sex (vs. female sex) } \\
\hline At 3 months & $0.6[0.4$ to 1.0$](P=.064)$ & $0.8[0.5$ to 1.5$](P=.58)$ \\
\hline At 12 months & $0.4[0.2$ to 0.7$](P=.002)^{*}$ & $0.4[0.2$ to 0.9$](P=.033)^{*}$ \\
\hline At 24 months & $0.7[0.4$ to 1.1$](P=.11)$ & $0.8[0.4$ to 1.5$](P=.49)$ \\
\hline At 36 months & $1.1[0.7$ to 1.8$](P=.77)$ & $1.4[0.7$ to 2.6$](P=.32)$ \\
\hline \multicolumn{3}{|c|}{ Ambulatory with aid before CLI onset (vs. ambulatory without aid) } \\
\hline At 3 months & $5.4[3.0$ to 9.8$](P<.001)^{*}$ & $2.3[1.0$ to 5.3$](P=.046)^{*}$ \\
\hline At 12 months & $3.8[2.0$ to 7.5$](P<.001)^{*}$ & $2.3[0.9$ to 5.8$](P=.083)$ \\
\hline At 24 months & $3.5[1.8$ to 6.9$](P<.001)^{*}$ & $2.1[0.7$ to 6.1$](P=.15)$ \\
\hline At 36 months & $3.1[1.4$ to 6.6$](P=.005)^{*}$ & $2.2[0.7$ to 6.4$](P=.16)$ \\
\hline \multicolumn{3}{|c|}{ Ambulatory with aid at revascularization (vs. ambulatory without aid) } \\
\hline At 3 months & $5.8[3.1$ to 10.8$](P<.001)^{*}$ & $3.5[1.5$ to 8.1$](P=.003)^{*}$ \\
\hline At 12 months & $2.7[1.5$ to 4.9$](P=.001)^{*}$ & $1.4[0.6$ to 3.1$](P=.47)$ \\
\hline At 24 months & $2.4[1.3$ to 4.5$](P=.005)^{*}$ & $1.6[0.6$ to 4.6$](P=.36)$ \\
\hline At 36 months & $2.1[1.1$ to 3.8$](P=.019)^{*}$ & $1.4[0.6$ to 3.6$](P=.44)$ \\
\hline \multicolumn{3}{|c|}{ Non-ambulatory at revascularization (vs. ambulatory without aid) } \\
\hline At 3 months & $9.5[5.3$ to 16.9$](P<.001)^{*}$ & $6.2[3.2$ to 12.1$](P<.001)^{*}$ \\
\hline At 12 months & $5.3[3.0$ to 9.2$](P<.001)^{*}$ & $3.8[2.0$ to 7.2$](P<.001)^{*}$ \\
\hline At 24 months & $7.2[3.5$ to 14.6$](P<.001)^{*}$ & $5.4[2.1$ to 14.1$](P=.001)^{*}$ \\
\hline At 36 months & $4.8[2.2$ to 10.5$](P<.001)^{*}$ & $3.6[1.4$ to 9.2$](P=.011)^{*}$ \\
\hline \multicolumn{3}{|l|}{ Current smoking } \\
\hline At 3 months & $0.6[0.3$ to 1.0$](P=.054)$ & $0.8[0.4$ to 1.7$](P=.60)$ \\
\hline At 12 months & $0.6[0.3$ to 1.1$](P=.096)$ & $1.1[0.5$ to 2.4$](P=.82)$ \\
\hline At 24 months & $0.5[0.3$ to 1.0$](P=.039)^{*}$ & $0.8[0.4$ to 2.0$](P=.68)$ \\
\hline At 36 months & $0.6[0.3$ to 1.1$](P=.13)$ & $0.9[0.5$ to 1.9$](P=.85)$ \\
\hline \multicolumn{3}{|l|}{ Diabetes mellitus } \\
\hline At 3 months & $1.5[0.9$ to 2.4$](P=.10)$ & $1.3[0.7$ to 2.3$](P=.40)$ \\
\hline At 12 months & $0.8[0.5$ to 1.5$](P=.55)$ & $0.7[0.3$ to 1.3$](P=.21)$ \\
\hline At 24 months & $1.8[1.1$ to 2.9$](P=.024)^{*}$ & $1.6[0.9$ to 3.0$](P=.099)$ \\
\hline At 36 months & $1.8[1.1$ to 3.2$](P=.031)^{*}$ & $1.6[0.9$ to 3.0$](P=.14)$ \\
\hline \multicolumn{3}{|c|}{ Renal failure without dialysis (vs eGFR $\geq 30 \mathrm{ml} / \mathrm{min} / 1.73 \mathrm{~m}^{2}$ ) } \\
\hline At 3 months & $2.1[0.8$ to 5.6$](P=.15)$ & $1.5[0.5$ to 4.7$](P=.49)$ \\
\hline At 12 months & $2.3[0.8$ to 6.6$](P=.13)$ & $2.5[0.7$ to 8.6$](P=.14)$ \\
\hline At 24 months & $1.7[0.5$ to 5.6$](P=.41)$ & $1.4[0.3$ to 5.8$](P=.66)$ \\
\hline At 36 months & $2.1[0.7$ to 6.8$](P=.21)$ & $1.5[0.4$ to 5.6$](P=.54)$ \\
\hline \multicolumn{3}{|c|}{ Renal failure on dialysis (vs eGFR $\geq 30 \mathrm{ml} / \mathrm{min} / 1.73 \mathrm{~m}^{2}$ ) } \\
\hline At 3 months & $2.1[1.3$ to 3.2$](P=.001)^{*}$ & $2.4[1.4$ to 4.2$](P=.001)^{*}$ \\
\hline At 12 months & $2.5[1.5$ to 4.2$](P=.001)^{*}$ & $3.3[1.6$ to 6.8$](P=.002)^{*}$ \\
\hline At 24 months & $3.3[2.1$ to 5.2$](P<.001)^{*}$ & $3.6[2.1$ to 6.3$](P<.001)^{*}$ \\
\hline At 36 months & $3.1[1.8$ to 5.2$](P<.001)^{*}$ & $2.7[1.6$ to 4.8$](P<.001)^{*}$ \\
\hline
\end{tabular}


(Cont. Supplementary Table 1)

\begin{tabular}{|c|c|c|}
\hline & Crude odds ratio & Adjusted odds ratio \\
\hline At 3 months & $2.1[1.1$ to 4.1$](P=.032)^{*}$ & $1.5[0.6$ to 3.4$](P=.36)$ \\
\hline At 24 months & $1.5[0.8$ to 2.9$](P=.18)$ & $0.9[0.4$ to 2.0$](P=.79)$ \\
\hline At 36 months & $1.8[0.9$ to 3.6$](P=.094)$ & $1.1[0.5$ to 2.4$](P=.82)$ \\
\hline At 12 months & $0.9[0.8$ to 1.0$](P=.020)^{*}$ & $1.0[0.9$ to 1.2$](P=.65)$ \\
\hline At 24 months & $0.8[0.7$ to 0.9$](P=.001)^{*}$ & $1.0[0.8$ to 1.1$](P=.73)$ \\
\hline At 36 months & $0.8[0.7$ to 0.9$](P=.001)^{*}$ & $0.9[0.8$ to 1.1$](P=.27)$ \\
\hline \multicolumn{3}{|c|}{ WIfI clinical stage 4 (vs. WIfI clinical stage 2/3) } \\
\hline At 36 months & $1.3[0.9$ to 2.1$](P=.21)$ & $1.2[0.7$ to 2.1$](P=.44)$ \\
\hline \multicolumn{3}{|c|}{ Surgical reconstruction (vs. EVT alone) } \\
\hline At 3 months & $1.1[0.7$ to 1.7$](P=.74)$ & $1.0[0.6$ to 1.7$](P=.89)$ \\
\hline At 12 months & $0.6[0.4$ to 1.0$](P=.041)^{*}$ & $0.5[0.3$ to 1.0$](P=.037)^{*}$ \\
\hline At 24 months & $0.6[0.4$ to 0.9$](P=.024)^{*}$ & $0.5[0.2$ to 0.9$](P=.022)^{*}$ \\
\hline At 36 months & $0.6[0.4$ to 1.0$](P=.054)$ & $0.5[0.3$ to 1.0$](P=.041)^{*}$ \\
\hline
\end{tabular}

Data are odds ratios for independent ambulation loss at 3, 12, 24, and 36 months [95\% confidence intervals] ( $P$ values). Crude odds ratios were derived from the univariate logistic regression model, whereas adjusted odds ratios were derived from the multivariate logistic regression model in which all the variables listed in the tables were entered as explanatory variables. Asterisks indicate $P<.05$. 
Supplementary Table 2. Associations of baseline characteristics with ambulation loss in patients who were non-ambulatory at revascularization

\begin{tabular}{lll}
\hline & Crude odds ratio & Adjusted odds ratio \\
\hline Age (per 10 years) & & $1.4[0.8$ to 2.3$](P=.23)$ \\
At 3 months & $1.2[0.8$ to 1.8$](P=.44)$ & $1.3[0.8$ to 2.3$](P=.32)$ \\
At 12 months & $1.1[0.7$ to 1.6$](P=.79)$ & $1.7[0.8$ to 3.6$](P=.16)$ \\
At 24 months & $1.3[0.7$ to 2.4$](P=.43)$ & $1.5[0.7$ to 3.0$](P=.30)$ \\
At 36 months & $0.9[0.5$ to 1.7$](P=.72)$ & $1.2[0.5$ to 3.4$](P=.67)$ \\
\hline Male sex (vs. female sex) & & $0.8[0.2$ to 2.5$](P=.66)$ \\
At 3 months & $0.9[0.4$ to 2.0$](P=.76)$ & $1.2[0.3$ to 5.1$](P=.76)$ \\
At 12 months & $0.6[0.2$ to 1.4$](P=.20)$ & $1.4[0.3$ to 7.6$](P=.69)$ \\
At 24 months & $0.8[0.3$ to 2.1$](P=.67)$ & $1.2[0.3$ to 4.7$](P=.80)$ \\
At 36 months & & \\
\hline
\end{tabular}

Ambulatory with aid before CLI onset (vs. ambulatory without aid)

At 3 months

At 12 months

At 24 months

At 36 months
2.9 [1.2 to 7.1$](P=.018)^{*}$

4.1 [1.5 to 11.2 ] $(P=.007)^{*}$

4.5 [1.1 to 18.0$](P=.034)^{*}$

3.3 [0.9 to 11.7$](P=.067)$
4.1 [1.4 to 12.4$](P=.012)^{*}$

8.2 [1.8 to 38.3] $(P=.008)^{*}$

$7.1[0.9$ to 53.4$](P=.057)$

7.5 [1.4 to 40.6$](P=.021)^{*}$

\section{Current smoking}

At 3 months

At 12 months

0.8 [0.2 to 3.9] $(P=.80)$

$0.5[0.1$ to 2.4$](P=.40)$

$1.4[0.2$ to 9.4$](P=.70)$

At 24 months

0.8 [0.1 to 4.2] $(P=.77)$

(not converged)

$0.9[0.1$ to 6.8$](P=.91)$

$1.7[0.2$ to 17.6$](P=.63)$

(not converged)

Diabetes mellitus

At 3 months

At 12 months

At 24 months

$1.2[0.4$ to 3.7$](P=.74)$

$1.3[0.4$ to 4.4$](P=.72)$

$0.9[0.3$ to 2.6$](P=.83)$

$0.7[0.2$ to 2.8$](P=.56)$

$1.0[0.3$ to 3.4$](P=.97)$

0.8 [0.2 to 3.9] $(P=.81)$

At 36 months

$1.3[0.4$ to 5.0$](P=.66)$

$1.2[0.2$ to 6.0$](P=.84)$

Renal failure without dialysis (vs eGFR $\geq 30 \mathrm{ml} / \mathrm{min} / 1.73 \mathrm{~m}^{2}$ )

At 3 months

At 12 months

At 24 months

At 36 months (not converged)

$0.5[<0.1$ to 5.8$](P=.56)$

$0.5[<0.1$ to 8.9$](P=.66)$

$1.0[<0.1$ to 12.9$](P=.97)$ (not converged)

3.5 [0.1 to 84.7$](P=.43)$

$2.6[0.1$ to 56.6$](P=.53)$

$4.6[0.2$ to 111$](P=.35)$

Renal failure on dialysis (vs eGFR $\geq 30 \mathrm{ml} / \mathrm{min} / 1.73 \mathrm{~m}^{2}$ )

At 3 months

At 12 months

$1.6[0.6$ to 4.0$](P=.30)$

$2.4[1.0$ to 5.6$](P=.042)^{*}$

$2.0[0.7$ to 5.7$](P=.19)$

At 24 months

$2.3[0.7$ to 7.1$](P=.15)$

At 36 months

3.4 [1.1 to 10.9 ] $(P=.041)^{*}$

4.2 [1.3 to 13.4$](P=.014)^{*}$

4.0 [1.0 to 15.9$](P=.050)^{*}$

7.3 [1.7 to 31.6] $(P=.009)^{*}$

Heart failure

At 3 months

At 12 months

$1.1[0.4$ to 2.8$](P=.85)$

$0.9[0.3$ to 3.0$](P=.91)$

At 24 months

$1.4[0.5$ to 3.8$](P=.48)$

0.9 [0.2 to 3.2] $(P=.83)$

At 36 months

$1.4[0.5$ to 4.3$](P=.54)$

0.9 [0.2 to 4.3] $(P=.94)$

2.4 [0.6 to 10.2$](P=.24)$

$2.0[0.4$ to 10.2$](P=.40)$

$\mathrm{Hb}$ (per $1 \mathrm{~g} / \mathrm{dl}$ )

At 3 months

At 12 months

$0.9[0.8$ to 1.2$](P=.63)$

$1.0[0.7$ to 1.3$](P=.77)$

1.0 [0.8 to 1.2$](P=.73)$

$1.1[0.8$ to 1.5$](P=.58)$

At 24 months

1.0 [0.8 to 1.3$](P=.94)$

$1.1[0.8$ to 1.4$](P=.70)$

At 36 months

1.0 [0.8 to 1.4$](P=.75)$ 
(Cont. Supplementary Table 2)

Crude odds ratio

WIfI clinical stage 4 (vs. WIfI clinical stage $2 / 3$ )

At 3 months

At 12 months

At 24 months

At 36 months

Surgical reconstruction (vs. EVT alone)

At 3 months

At 12 months

At 24 months

At 36 months

$$
\begin{array}{ll}
2.0[0.9 \text { to } 4.5](P=.095) & 3.2[1.1 \text { to } 9.1](P=.027)^{*} \\
2.6[1.1 \text { to } 6.1](P=.023)^{*} & 7.6[1.7 \text { to } 33.2](P=.009)^{*} \\
1.5[0.6 \text { to } 4.0](P=.41) & 3.8[0.8 \text { to } 18.7](P=.092) \\
1.9[0.7 \text { to } 4.9](P=.19) & 5.0[1.2 \text { to } 20.9](P=.029)^{*}
\end{array}
$$

$$
\begin{array}{ll}
0.8[0.3 \text { to } 1.7](P=.49) & 0.9[0.4 \text { to } 2.2](P=.81) \\
0.5[0.2 \text { to } 1.2](P=.14) & 0.6[0.2 \text { to } 1.8](P=.34) \\
0.4[0.2 \text { to } 1.0](P=.051) & 0.5[0.1 \text { to } 1.5](P=.20) \\
0.5[0.2 \text { to } 1.7](P=.28) & 0.7[0.2 \text { to } 2.6](P=.53)
\end{array}
$$

Data are odds ratios for ambulation loss (regardless of aid) at 3, 12, 24, and 36 months [95\% confidence intervals] ( $P$ values). Crude odds ratios were derived from the univariate logistic regression model, whereas adjusted odds ratios were derived from the multivariate logistic regression model in which all the variables listed in the tables were entered as explanatory variables. Asterisks indicate $P<.05$. 

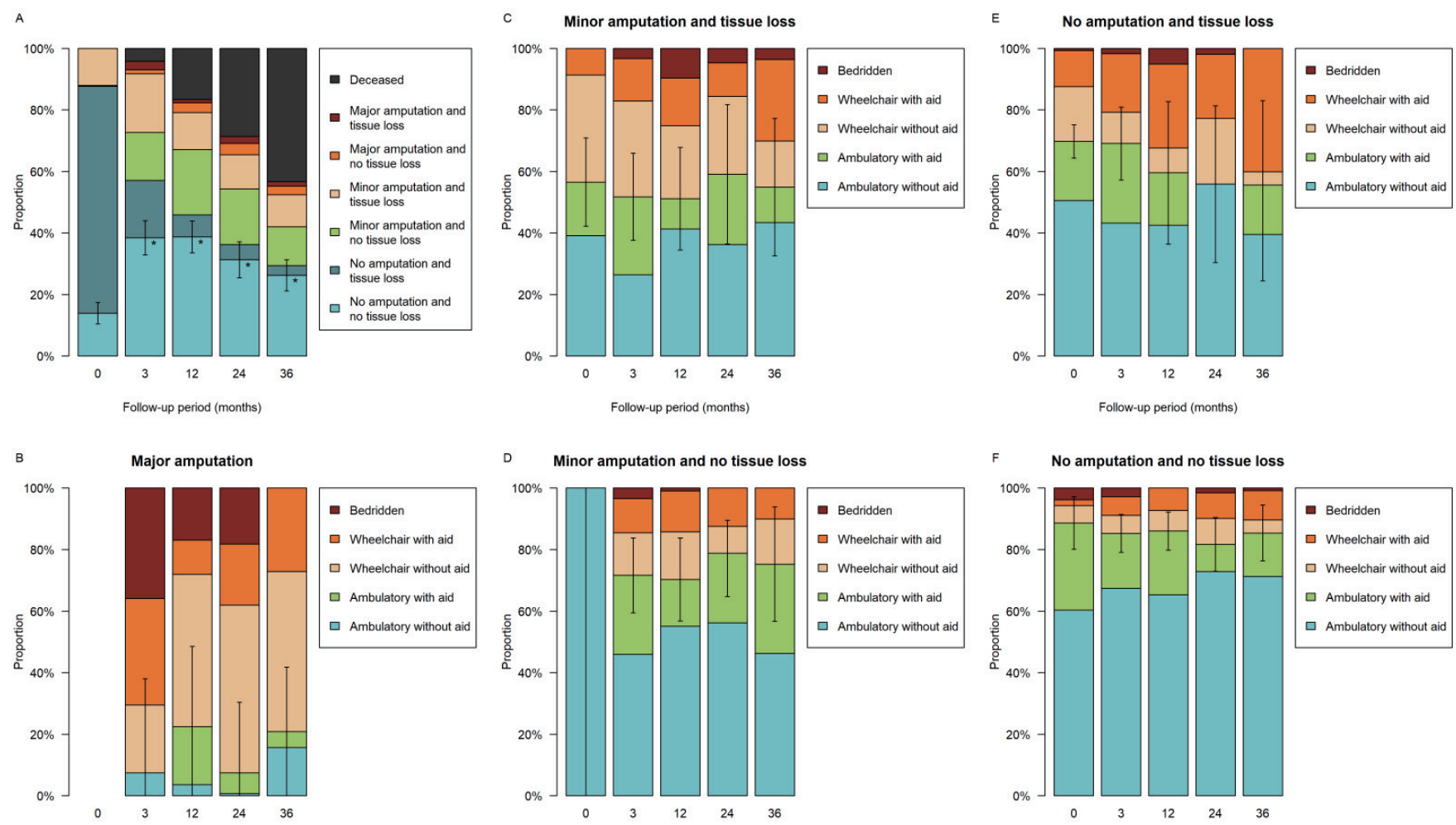

Supplementary Fig. 2. Limb status and ambulation

Panels A illustrate the proportions of the respective limb statuses in the overall population. Error bars indicate $95 \%$ confidence intervals for the proportion of patients without amputation or tissue loss, and asterisks represent $P<.05$ versus the baseline value regarding the proportion of patients without amputation or tissue loss. Panels B to F illustrate the proportions of respective mobility statuses in patients alive with major amputation (regardless of tissue loss) at each time point (B), in those alive with minor amputation and tissue loss at each time point $(\mathrm{C})$, in those alive with minor amputation and no tissue loss at each time point (D), in those alive with no amputation and tissue loss at each time point (E), and in those alive with no amputation and no tissue loss at each time point (F). Note that in Panel B, patients alive with major amputation were not distinguished by the presence of tissue loss, simply because of their small number. Error bars in Panels B to F indicate 95\% confidence intervals for the proportion of patients who were ambulatory regardless of aid.

Supplementary Table 3. Associations of limb status at a specific time point with ambulation loss at the same time point

\begin{tabular}{|c|c|c|c|c|}
\hline & At 3 months & At 12 months & At 24 months & At 36 months \\
\hline Tissue loss & $2.5[1.5$ to 4.1$](P<.001)^{*}$ & $2.9[1.5$ to 5.6$](P=.001)^{*}$ & $2.6[1.0$ to 7.3$](P=.060)$ & $3.0[1.0$ to 8.7$](P=.044)^{*}$ \\
\hline \multicolumn{5}{|l|}{ Amputation (vs. none) } \\
\hline Minor amputation & $2.2[1.3$ to 3.7$](P=.004)^{*}$ & $2.2[1.1$ to 4.1$](P=.020)^{*}$ & $1.2[0.6$ to 2.5$](P=.64)$ & $1.6[0.7$ to 3.8$](P=.26)$ \\
\hline Major amputation & (Not converged) & $15.8[2.4$ to 104$](P=.007)^{*}$ & (Not converged) & $16.3[2.9$ to 90.8$](P=.002)^{*}$ \\
\hline
\end{tabular}

Data are multivariate odds ratios for ambulation loss (regardless of aid) at 3, 12, 24, and 36 months [95\% confidence intervals] ( $P$ values), which were derived from the multivariate logistic regression model in which tissue loss and amputation at the index time point were entered as explanatory variables. Asterisks indicate $P<.05$. 